\title{
Dynamical solution to the axion domain wall problem
}

\author{
Masahiro Ibe, ${ }^{1,3,{ }^{*}}$ Shin Kobayashi, ${ }^{1, \dagger}$ Motoo Suzuki ${ }^{2,1, \hbar}$ and Tsutomu T. Yanagida ${ }^{2,3,8}$ \\ ${ }^{1}$ ICRR, The University of Tokyo, Kashiwa, Chiba 277-8582, Japan \\ ${ }^{2}$ T. D. Lee Institute and School of Physics and Astronomy, Shanghai Jiao Tong University, \\ Shanghai 200240, China \\ ${ }^{3}$ Kavli IPMU (WPI), UTIAS, The University of Tokyo, Kashiwa, Chiba 277-8583, Japan
}

(Received 3 October 2019; accepted 27 January 2020; published 21 February 2020)

\begin{abstract}
The domain wall problem and the isocurvature problem restrict possible combinations of axion models and inflation models. In this paper, we consider a new mechanism which solves those problems by dynamics of multiple scalar fields during/after inflation. The mechanism makes axion models with a nontrivial domain wall number compatible with inflation models with a large Hubble parameter, $H_{I} \gg 10^{7-8} \mathrm{GeV}$. The isocurvature problem is also avoided in the mechanism.
\end{abstract}

DOI: 10.1103/PhysRevD.101.035029

\section{INTRODUCTION}

The Peccei-Quinn (PQ) mechanism is the most plausible solution to the strong $(C P)$ problem [1,2]. In this mechanism, the effective $\theta$ angle of QCD is canceled by the vacuum expectation value (VEV) of the pseudo-NambuGoldstone boson, axion $a$, which is associated with the spontaneous breaking of the global $U(1)$ symmetry (PQ symmetry) $[3,4]$. The mechanism is particularly attractive, as the invisible axion [5-8] is a good candidate for cold dark matter [9-12]. The physics of the axion depends on when the PQ symmetry is broken in the history of the Universe.

In the case where the PQ symmetry is spontaneously broken after the end of inflation, too many domain walls are produced, and most models are excluded except for the so-called domain wall number $N_{\mathrm{DW}}=1$ scenario (see, e.g., $[13,14])$. For $N_{\mathrm{DW}}=1$, the string-wall network collapses immediately after the QCD phase transition while emitting axions [15]. The resultant axions can be the dominant dark matter component for $f_{a} \simeq 5 \times 10^{10}$ according to lattice simulations $[16,17]$. This kind of scenario is possible in the Kim-Shifman-Vainshtein-Zakharov (KSVZ) model [5,6] and can be distinguished by the axion search experiments (see, e.g., [18]). For $N_{\mathrm{DW}}>1$, the domain wall is stable and dominates the Universe eventually. This domain wall

\footnotetext{
*ibe@icrr.u-tokyo.ac.jp

†shinkoba@icrr.u-tokyo.ac.jp

*m0t@icrr.u-tokyo.ac.jp

\$tsutomu.tyanagida@ipmu.jp
}

Published by the American Physical Society under the terms of the Creative Commons Attribution 4.0 International license. Further distribution of this work must maintain attribution to the author(s) and the published article's title, journal citation, and DOI. Funded by SCOAP. problem can be avoided if the PQ symmetry is explicitly broken. ${ }^{1}$ Then, the string-wall network is not exactly stable even for $N_{\mathrm{DW}}>1$. However, the explicit breaking, which is required to make the string-wall network collapse fast enough, encounters the strong $C P$ problem again [23,24].

If the PQ symmetry is broken before/during inflation, no domain wall is formed below the QCD scale even in the case $N_{\text {DW }}>1$. However, the so-called isocurvature problem arises [25-30]. From the observation of the cosmic microwave background [31], the isocurvature perturbation produced by the quantum fluctuation of the axion is constrained. It requires a small inflation scale less than $10^{7-8} \mathrm{GeV}$ to suppress the fluctuation (see, e.g., $[13,32]$ ). In this scenario, the axion is produced by the so-called misalignment mechanism, which can be tested by ongoing [33] and future experiments (see, e.g., [34-36]).

In this paper, we discuss a new mechanism which solves those problems by dynamics of multiple scalar fields during/after inflation. This mechanism enables the axion model with $f_{a} \sim 10^{12} \mathrm{GeV}$ to be compatible with the models of inflation with $H_{I} \sim 10^{13} \mathrm{GeV}$. Such a large axion decay constant and the large Hubble parameter during inflation can be tested by the axion search experiments (see, e.g., [18]) and the searches for the primordial $B$-mode polarization in the cosmic microwave background (CMB) (see, e.g., [37]), respectively.

In the new mechanism, we may consider any type of axion model. We call the PQ charged field, which spontaneously breaks the PQ symmetry, the PQ field. Then, we introduce an additional PQ charged scalar field which obtains a vanishing VEV. We call this additional field the

\footnotetext{
${ }^{1}$ Other approaches such as introducing a new confinement sector or piercing the wall with primordial black holes can be found in Refs. [19-22].
} 
spectator PQ field. We assume that the spectator PQ field obtains a large field value during/after inflation. The large field value of the spectator PQ field provides a nontrivial scalar potential of the axion when the PQ field obtains its VEV after inflation. The nontrivial axion potential prohibits the formation of the cosmic string, and hence, prohibits the string-wall network below the QCD scale. The isocurvature perturbation, which stems from the quantum fluctuation of the spectator PQ field, is suppressed by its large field value during inflation [38]. In this way, the new mechanism solves the domain wall problem without causing the isocurvature problem. This mechanism may be regarded as a multifield version of the mechanism discussed in [39]. ${ }^{2}$

The organization of the paper is as follows. In Sec. II, we summarize the setup of our model. In Sec. III, we discuss how the spectator PQ field evolves. In Sec. IV, we discuss how the axion behaves in the presence of the spectator PQ field. We also discuss the viable parameter region of the new mechanism. In Sec. V, we discuss the supersymmetric extension of the model. The final section is devoted to our conclusions.

\section{PECCEI-QUINN MECHANISM WITH A SPECTATOR PQ FIELD}

\section{A. General recipe of the dynamical solution}

Before discussing the details of the mechanism, we summarize the general recipe for the dynamical solution of the domain wall and the isocurvature problems.

(1) Bring an axion model where the PQ symmetry is spontaneously broken by the VEV of the PQ field $P$.

(2) Add a spectator PQ field $S$, which obtains a vanishing VEV but has a large field value in the early Universe until $P$ obtains the VEV (Sec. III).

(3) Introduce a mixing term between $P$ and $S$ so that $P$ feels a strong PQ symmetry breaking effect when it obtains the VEV (Sec. IV).

(4) Make the effects of the mixing term inefficient before $S$ starts coherent oscillation around its origin (Sec. IV).

With the large field value of $S, P$ feels a strong PQ symmetry breaking, and no cosmic strings are formed when $P$ obtains the VEV. The fourth condition is important not to randomize the axion field value even after $S$ starts the coherent oscillation (Sec. IV C). Without cosmic strings and with the uniform axion field value, the domain walls are not formed after the QCD phase transition. The quantum fluctuation of the phase component of $S$ is imprinted in the axion through the mixing term. The isocurvature problem can be avoided by requiring

\footnotetext{
${ }^{2}$ See also [40-43] for other realization of dynamics which solves the domain wall and the isocurvature problems. The solutions in the context of the axion predicted in string theory [44-46] have also been discussed, where the axion dark matter abundance is suppressed dynamically $[47,48]$.
}

that the field value of $S$ is of $\mathcal{O}\left(M_{\mathrm{Pl}}\right)$ during inflation (Sec. IV D). In any successful implementation of this mechanism, the domain wall and the isocurvature problems are solved dynamically.

\section{B. KSVZ axion model}

As a concrete example of the axion model, we consider the KSVZ axion model $[5,6]$ in which the PQ field $P$ obtains the VEV via the scalar potential,

$$
V(P)=\lambda_{p}\left(|P|^{2}-\frac{v_{\mathrm{PQ}}^{2}}{2}\right)^{2}
$$

Here, $\lambda_{p}$ is a coupling constant of $\mathcal{O}(1)$, and $v_{\mathrm{PQ}}$ is a parameter with mass dimension. The VEV of the PQ field is given by $\langle P\rangle=v_{\mathrm{PQ}} / \sqrt{2}$. The axion field $a$ corresponds to the phase component of $P$,

$$
P=\frac{1}{\sqrt{2}} v_{\mathrm{PQ}} e^{i a / v_{\mathrm{PQ}}}
$$

where we omit the radial component of $P$ for brevity.

The PQ field couples to the $N_{f}$ vectorlike quarks in the fundamental representation of the $S U(3)$ gauge group of QCD $\left(Q_{L}, \bar{Q}_{R}\right)$ via

$$
\mathcal{L}=y_{\mathrm{KSVZ}} P Q_{L} \bar{Q}_{R}+\text { H.c. },
$$

with $y_{\mathrm{KSVZ}}$ being the coupling constant. Below the mass scale of the KSVZ quarks $y_{\mathrm{KSVZ}} v_{\mathrm{PQ}}$, the QCD anomaly induces the axion couplings to $\mathrm{QCD}$,

$$
L=\frac{g_{s}^{2}}{32 \pi^{2}} \frac{N_{f}}{v_{\mathrm{P} Q}} a G \tilde{G} .
$$

Here, $g_{s}$ denotes the QCD gauge coupling constant, and $G$ and $\tilde{G}$ are the QCD field strength and its Hodge dual, respectively. The Lorentz and color indices are understood. We define the origin of the axion field space at which the effective $\theta$ angle of QCD is vanishing.

Below the QCD scale, the above interaction term in Eq. (4) leads to the scalar potential of the axion,

$$
V(a) \sim m_{a}^{2} f_{a}^{2}\left[1-\cos \frac{a}{f_{a}}\right] .
$$

Here, $f_{a}=v_{\mathrm{PQ}} / N_{f}$ is the effective decay constant of the axion, and $m_{a}$ denotes the mass of the axion which is estimated to be

$$
m_{a} \simeq 6 \mu \mathrm{eV}\left(\frac{10^{12} \mathrm{GeV}}{f_{a}}\right)
$$


(see, e.g., $[49,50])$. It should be noted that the domain of the axion field is given by $a / f_{a}=\left[0,2 \pi N_{f}\right)$, and hence, $N_{f}$ corresponds to the domain wall number $N_{\text {DW }}=N_{f}$.

As we will see below, the axion field value settles to a nonzero value $a_{i}$ of $\mathcal{O}\left(f_{a}\right)$ after a complex dynamics of the new mechanism. Below the QCD scale, the axion starts coherent oscillation from the nonzero field value around its origin which behaves as cold dark matter as in the conventional misalignment mechanism [9-11]. The axion dark matter density is given by [51]

$$
\Omega_{a} h^{2} \simeq 0.2 \times\left(\frac{a_{i}}{f_{a}}\right)^{2}\left(\frac{f_{a}}{10^{12} \mathrm{GeV}}\right)^{1.19} .
$$

Based on this estimate, we focus on the case with $f_{a}=$ $\mathcal{O}\left(10^{12}\right) \mathrm{GeV}$ in the following discussion.

\section{Spectator PQ field}

Now let us introduce another PQ charged scalar field, the spectator PQ field $S$. We assume that $S$ has a PQ charge which is $-1 / m$ of that of the PQ field $(m \in \mathbb{N})$. With this assumption, $S$ couples to $P$ via

$$
\begin{aligned}
V(P, S)= & \lambda_{p}\left(|P|^{2}-\frac{v_{\mathrm{PQ}}^{2}}{2}\right)^{2}+m_{S}^{2}|S|^{2}+\frac{1}{(n !)^{2}} \frac{\lambda_{s}^{2}}{M_{\mathrm{Pl}}^{2 n-4}}|S|^{2 n} \\
& +\frac{\lambda}{m ! M_{\mathrm{Pl}}^{m-3}} S^{m} P+\text { H.c. }
\end{aligned}
$$

Here, $m_{S}$ is the mass parameter of $S, \lambda_{s}$ and $\lambda$ are dimensionless coupling constants, and $M_{\mathrm{Pl}} \simeq 2.4 \times 10^{18} \mathrm{GeV}$ is the reduced Planck scale. Because of the positive mass squared, $S$ does not obtain a nonvanishing VEV. As we will see in the following two sections, $n$ is required to be larger than 5 for a successful mechanism. The absence of the scalar potential terms of $S$ with a lower dimension than that of $|S|^{2 n}$ will be justified in the supersymmetric extension discussed in Sec. V.

In this mechanism, a large field value of $S$ during/after inflation plays a crucial role in solving the domain wall problem and the isocurvature problem. For that purpose, we introduce interactions between the (spectator) PQ fields with the inflaton field $\phi$,

$$
\begin{aligned}
V(P, S, \phi)= & V(\phi)+V(P, S)+\frac{c_{p}}{3} \frac{V(\phi)}{M_{\mathrm{Pl}}^{2}}|P|^{2} \\
& -\frac{c_{s}}{3} \frac{V(\phi)}{M_{\mathrm{Pl}}^{2}}|S|^{2},
\end{aligned}
$$

where $c_{p}$ and $c_{s}$ are positive valued coupling constants. $V(\phi)$ denotes the inflaton potential with which the Hubble parameter during inflation is given by

$$
H_{I}^{2}=\frac{V(\phi)}{3 M_{\mathrm{Pl}}^{2}} .
$$

Through the interactions with the inflaton, $P$ and $S$ obtain the positive and negative Hubble-induced mass terms during inflation,

$$
\tilde{m}_{P}^{2}=c_{p} H_{I}^{2}(>0), \quad \tilde{m}_{S}^{2}=-c_{S} H_{I}^{2}(<0),
$$

respectively. Generally, scalar fields obtain Hubble-induced mass terms. We also discuss how the interactions with the inflaton in Eq. (9) can be obtained in the supersymmetric extension.

In the following scenario, we assume that $S$ is never in thermal equilibrium. Such a situation can be easily realized when the inflaton field mainly decays into the Standard Model particles. Late-time interactions of $S$ with thermal bath particles are negligible, as it only couples to other fields through the Planck suppressed operators.

\section{DYNAMICS OF THE SPECTATOR PQ FIELD}

\section{A. Inflation era}

During inflation, $S$ obtains a negative Hubble-induced mass term in Eq. (11), which is much larger than $m_{S}^{2}$ in size. Thus, the potential of $S$ can be approximated by

$$
V(S)=\frac{\lambda_{s}^{2}}{(n !)^{2} M_{\mathrm{Pl}}^{2 n-4}}|S|^{2 n}-c_{s} H_{I}^{2}|S|^{2} .
$$

Because of the Hubble-induced mass term, $S$ obtains a large expectation value,

$$
\left\langle S_{I}\right\rangle \simeq\left(\sqrt{\frac{c_{s}}{n}} \frac{n !}{\lambda_{s}}\right)^{\frac{1}{n-1}}\left(\frac{H_{I}}{M_{\mathrm{Pl}}}\right)^{\frac{1}{n-1}} M_{\mathrm{Pl}}
$$

Hereafter, the expectation value $\langle S\rangle$ denotes its absolute value if not otherwise specified. In the following analysis, we assume

$$
\lambda_{s} \gtrsim n ! \sqrt{\frac{c_{s}}{n}}\left(\frac{H_{I}}{M_{\mathrm{Pl}}}\right)
$$

so that $\left\langle S_{I}\right\rangle$ not to exceed $\mathcal{O}\left(M_{\mathrm{Pl}}\right)$. For example, $\left\langle S_{I}\right\rangle \sim M_{\mathrm{Pl}}$ for $n=6, H_{I}=10^{12} \mathrm{GeV}, c_{s}=1$, and $\lambda_{s}=10^{-4}$.

\section{B. Inflaton oscillation era}

After inflation, the inflaton starts coherent oscillation around its minimum. As the inflaton oscillation timescale becomes much shorter than the Hubble time, the dynamics of $S$ can be analyzed by taking the time average of the inflaton oscillation. Thus, the inflaton potential in Eq. (9) can be approximated by 


$$
\overline{V(\phi)}=\frac{3}{2} H^{2} M_{\mathrm{Pl}}^{2},
$$

where the bar denotes the time average, and $H$ is the Hubble parameter at that time. ${ }^{3}$

Now, let us focus on the dynamics of the radial component of $S$,

$$
S=\frac{\chi}{\sqrt{2}}, \quad(\chi \in \mathbb{R}) .
$$

Because of the PQ symmetry, the potential of $S$ does not induce the torque in the complex plane of $S$. Thus, the motion of $S$ is confined on a straight line passing through $S=0$ (see, e.g., Fig. 2). We will discuss how the phase component of $S$ behaves in the next section. In the above approximation, the equation of motion (EOM) of the zero mode of $\chi$ is given by

$$
\ddot{\chi}+3 H \dot{\chi}+\frac{n \lambda_{s}^{2}}{2^{n-1}(n !)^{2} M_{\mathrm{Pl}}^{2 n-4}} \chi^{2 n-1}-\frac{c_{s}}{2} H^{2} \chi=0,
$$

where the dot denotes the time derivative. We neglect $m_{s}^{2}$ by assuming that it is still much smaller than $H^{2}$ in this period.

Following [39], we introduce the $e$-folding number $N \equiv$ $\ln R$ as a time variable, where $R$ is the scale factor of the Universe. We define $R$ such that $R=1$ when the inflaton starts to oscillate. With the $e$-folding number, the EOM is rewritten by

$$
\frac{\mathrm{d}^{2}}{\mathrm{~d} N^{2}} \chi+\frac{3}{2} \frac{\mathrm{d}}{\mathrm{d} N} \chi+\frac{n \lambda_{s}^{2}}{2^{n-1}(n !)^{2} M_{\mathrm{Pl}}^{2 n-4} H^{2}} \chi^{2 n-1}-\frac{c_{s}}{2} \chi=0 .
$$

Next, we set $\chi$ in the form of

$\chi=\sigma \sqrt{2} M_{\mathrm{Pl}}\left(\frac{c_{s}(n !)^{2} H_{i}^{2}}{2 n \lambda_{s}^{2} M_{\mathrm{Pl}}^{2}}\right)^{\frac{1}{2(n-1)}} \exp \left[-\frac{3 N}{2(n-1)}\right]$.

Here, $H_{i}$ denotes the Hubble parameter at the onset of the inflaton oscillation. This leads to the EOM of $\sigma$,

$$
\begin{gathered}
\frac{\mathrm{d}^{2}}{\mathrm{~d} N^{2}} \sigma+\frac{3(n-3)}{2(n-1)} \frac{\mathrm{d}}{\mathrm{d} N} \sigma+\frac{c_{s}}{2} \sigma^{2 n-1} \\
-\left(\frac{9(n-2)}{4(n-1)^{2}}+\frac{c_{s}}{2}\right) \sigma=0 .
\end{gathered}
$$

The EOM of $\sigma$ represents a motion of a particle in a potential,

\footnotetext{
${ }^{3}$ At the beginning of the inflaton oscillation, in the case of chaotic inflation, the timescale of the inflaton oscillation is comparable to the Hubble time. However, this does not change the dynamics of $S$ significantly.
}

$$
V(\sigma)=-\frac{1}{2}\left(\frac{9(n-2)}{4(n-1)^{2}}+\frac{c_{s}}{2}\right) \sigma^{2}+\frac{c_{s}}{4 n} \sigma^{2 n}
$$

which has a minimum at

$$
\sigma_{0}=\left(1+\frac{9(n-2)}{2(n-1)^{2} c_{s}}\right)^{\frac{1}{2(n-1)}}
$$

The second term of the EOM is a velocity-dependent force. The initial position of $\sigma$ is given by Eq. (13),

$$
\sigma_{i} \sim 2^{\frac{1}{2(n-1)}},
$$

which is close to $\sigma_{0}$ for $c_{s}=\mathcal{O}(1)$.

The velocity-dependent force in Eq. (20) plays the role of friction for $n \geq 4$ (see also [39]). Thus, for $n \geq 4, \sigma$ which starts oscillation from $\sigma_{0}$ immediately settles down to $\sigma_{0}$ and stays there during the inflaton oscillation era. In this case, the field value of $S$ is roughly given by

$$
\langle S\rangle \simeq\left(\sqrt{\frac{c_{s}}{n}} \frac{n !}{\lambda_{s}}\right)^{\frac{1}{n-1}}\left(\frac{H}{M_{\mathrm{Pl}}}\right)^{\frac{1}{n-1}} M_{\mathrm{Pl}},
$$

which has the same dependence on the Hubble parameter in Eq. (13). This behavior of the scalar field is called the scaling solution [52].

For $n=3, \sigma$ keeps oscillating around $\sigma_{0}$ but does not go over $\sigma=0$ [39]. Thus, in this case, $S$ again keeps a large field value during the inflaton oscillation era. ${ }^{4}$ As pointed out in [53], however, the scalar field shows a peculiar behavior for $n=3$, the pseudoscaling solution, in which the field value gradually decreases in a zigzag manner.

For $n<3$, the velocity-dependent force accelerates the motion of $\sigma$, and eventually, the oscillation of $S$ goes over $S=0$, which results in $\langle S\rangle=0$. For a successful solution to the domain wall problem, we require that $S$ has a large field value when $P$ obtains the VEV in the radiationdominated (RD) era. Thus, we at least require $n \geq 3$ so that $S$ keeps a large field value during the inflaton oscillating period. As we will immediately see, however, we eventually require $n \geq 5$ for $S$ to have a large field value in the $\mathrm{RD}$ era (see also [52]).

\section{Radiation-dominated era}

In the RD era, the time dependence of the Hubble parameter changes from that in the inflaton oscillation era. In addition, the PQ fields no longer obtain the Hubbleinduced mass terms through the interactions with the inflaton.

In this mechanism, we assume that $m_{s}$ is much smaller than $H$ at the beginning of the RD era. Then, the EOM of $S$ can be written as

\footnotetext{
${ }^{4}$ The parametric resonance due to the oscillation of $S$ is not effective [39].
} 


$$
\ddot{\chi}+3 H \dot{\chi}+\frac{n \lambda_{s}^{2}}{2^{n-1}(n !)^{2} M_{\mathrm{Pl}}^{2 n-4}} \chi^{2 n-1}=0 .
$$

As in the inflaton oscillation era, we introduce the $e$-folding number $N \equiv \ln R$ which is vanishing at the beginning of the RD era. By using the $e$-folding number, the EOM is rewritten by

$$
\frac{\mathrm{d}^{2}}{\mathrm{~d} N^{2}} \sigma+\frac{n-5}{n-1} \frac{\mathrm{d}}{\mathrm{d} N} \sigma+\sigma^{2 n-1}-\frac{2(n-3)}{(n-1)^{2}} \sigma=0 .
$$

Here, we set

$$
\chi=\sigma \sqrt{2} M_{\mathrm{Pl}}\left(\frac{(n !)^{2} H_{r, i}^{2}}{n \lambda_{s}^{2} M_{\mathrm{Pl}}^{2}}\right)^{\frac{1}{2(n-1)}} \exp \left[-\frac{2 N}{n-1}\right],
$$

with $H_{r, i}$ being the Hubble parameter at the beginning of the RD era.

As in the case of the inflaton oscillation era, the EOM corresponds to a participle motion in a potential

$$
V(\sigma)=-\frac{(n-3)}{(n-1)^{2}} \sigma^{2}+\frac{1}{2 n} \sigma^{2 n},
$$

which has the minimum at

$$
\sigma_{r, 0}=\left[\frac{2(n-3)}{(n-1)^{2}}\right]^{\frac{1}{2(n-1)}}
$$

The initial position of $\sigma$ is roughly given by

$$
\sigma_{r, i} \sim c_{s}^{\frac{1}{2(n-1)}} .
$$

Similar to the case of the inflaton oscillation era, the friction term has a wrong sign for $n \leq 4$. For $n>5$, on the other hand, $S$ again behaves as the scaling solution [52],

$$
\begin{aligned}
\langle S\rangle & \simeq\left[\frac{2(n-3)(n !)^{2}}{n(n-1)^{2} \lambda_{s}^{2}}\right]^{\frac{1}{2(n-1)}}\left(\frac{H}{M_{\mathrm{Pl}}}\right)^{\frac{1}{n-1}} M_{\mathrm{Pl}} \\
& \simeq\left(\frac{n !}{\lambda_{s}}\right)^{\frac{1}{n-1}}\left(\frac{H}{M_{\mathrm{Pl}}}\right)^{\frac{1}{n-1}} M_{\mathrm{Pl}} .
\end{aligned}
$$

For $n=5, S$ behaves as the pseudosolution [53]. In summary, $S$ keeps a large field value during the RD era for $n \geq 5$. In the following analysis, we take $n=6$ as the minimal model, since the zigzag behavior in the pseudoscaling solution makes the analysis complicated.

While $H \gtrsim m_{S}, S$ obeys the scaling solution. ${ }^{5}$

When the Hubble parameter decreases further and becomes smaller than $m_{s}, S$ finally starts to oscillate around

\footnotetext{
${ }^{5}$ When $S$ obeys the scaling solution, $S$ does not lose its potential energy through particle emission.
}

its origin. For $n=6, \lambda_{s}=10^{-4}$, and $m_{S} \lesssim 10^{-15} \mathrm{GeV}$, the contribution of the coherent oscillation of $S$ to the DM abundance is negligibly small. ${ }^{6}$ For a larger $m_{S}$, we assume that $S$ decays into massless fermions $\psi_{s}$ through

$$
\mathcal{L}_{S \text {-decay }}=-y_{s} S \bar{\psi}_{s} \psi_{s}+\text { H.c. }
$$

With this assumption, the energy density of $S$ does not cause any cosmological problem. The number density of the massless fermions is also negligibly small and does not contribute to dark radiation (see the Appendix).

As we will see below, the behavior of $S$ discussed in this section successfully solves the domain wall problem and the isocurvature problem.

\section{DYNAMICS OF THE AXION AND CONSTRAINTS}

Now, let us consider the dynamics of the PQ field and the axion. A notable feature of this mechanism is that the large field value of $S$ provides a nonvanishing effective linear term of $P$ through the $P-S$ mixing term [see Eq. (9)].

\section{A. Before PQ breaking}

During inflation, the minimum of the scalar potential of $P$ is shifted due to the effective linear term, and $P$ also obtains a nonzero field value which is determined by balancing between the quartic term $\lambda_{p}|P|^{4}$ and the effective linear term $\lambda\langle S\rangle^{m} P$, which is given by

$$
\begin{aligned}
\left\langle P_{I}\right\rangle & \sim M_{\mathrm{Pl}}\left(\frac{\lambda}{m ! \lambda_{p}}\right)^{1 / 3}\left(\frac{\left\langle S_{I}\right\rangle}{M_{\mathrm{Pl}}}\right)^{m / 3} \\
& \simeq M_{\mathrm{Pl}}\left[\frac{\lambda}{m ! \lambda_{p}}\left(\frac{n !}{\lambda_{s}}\right)^{\frac{m}{n-1}}\right]^{1 / 3}\left(\frac{H_{I}}{M_{\mathrm{Pl}}}\right)^{\frac{m}{3(n-1)}} .
\end{aligned}
$$

As we will see in the following, the minimal model which successfully solves the domain wall problem is the one with $n=6, m=11$. In this case, $\left\langle P_{I}\right\rangle$ is rather large during inflation,

$$
\left\langle P_{I}\right\rangle \sim 10^{14} \mathrm{GeV},
$$

where the parameters are set to $\lambda_{p}=1, \lambda_{s}=10^{-4}$, $\lambda=10^{-6}$, and $H_{I}=10^{12} \mathrm{GeV}$ as a benchmark point (see Sec. IV E). Accordingly, the KSVZ quarks obtain heavy masses through the Yukawa coupling in Eq. (3)

$$
M_{K S V Z} \simeq y_{\mathrm{KSVZ}}\left\langle P_{I}\right\rangle
$$

during inflation.

\footnotetext{
${ }^{6}$ For $m_{S}=\mathcal{O}\left(10^{-15}\right) \mathrm{GeV}$, the coherent oscillation of $S$ can be the dominant component of DM. Interestingly, this case provides an ultralight DM model whose initial condition of the coherent oscillation is dynamically determined.
} 
In the inflaton oscillation era, on the other hand, $S$ decreases as the scaling solution, and hence, the minimum of the potential of $P$ also decreases. By the time of the completion of the reheating process, the minimum position of $P$ becomes much smaller than the temperature of the Universe $T{ }^{7}$ Therefore, the KSVZ quarks and $P$ are thermalized by the completion of the reheating process. Once the KSVZ quarks and $P$ are thermalized, $P$ obtains a thermal potential. ${ }^{8}$

Because of the thermal mass of $P$ of $\mathcal{O}\left(T^{2}\right),{ }^{9} P$ is settled to

$$
\langle P\rangle \sim \frac{\lambda}{m ! T^{2} M_{\mathrm{Pl}}^{m-3}}\langle S\rangle^{m} \simeq \frac{\lambda}{m !}\left(\frac{n !}{\lambda_{s}}\right)^{\frac{m}{n-1}}\left(\frac{T}{M_{\mathrm{Pl}}}\right)^{\frac{2 m}{n-1}-2} M_{\mathrm{Pl}}
$$

This expectation value is much smaller than $v_{\mathrm{PQ}}=$ $\mathcal{O}\left(10^{12}\right) \mathrm{GeV}$. Thus, we can consider that $P$ is stabilized at the origin by the thermal mass term at the beginning of the RD era.

\section{B. After PQ breaking}

When $P$ obtains the VEV $\langle P\rangle=v_{\mathrm{PQ}} / \sqrt{2}$, the axion rolls down from the hilltop of the potential. The direction of the axion is random in each Hubble patch (see Fig. 1), which results in the formation of the cosmic strings. However, the axion settles down to its minimum of the cosine potential induced by the $P-S$ mixing term, which forms the domain walls around the strings. This situation is analogous to the string-wall network formation below the QCD scale in the $N_{\text {DW }}=1$ scenario. In the $N_{\text {DW }}=1$ scenario, the stringwall network collapses by itself when the energy of a domain wall exceeds that of a string [15].

The condition for the collapse of the string-wall network is given by

$$
\frac{\sigma_{\mathrm{w}} d_{H}^{2}}{T_{\mathrm{s}} d_{H}}>1
$$

where $\sigma_{\mathrm{w}}$ is the surface tension of the domain wall, $T_{\mathrm{s}}$ the tension of the string, and $d_{H} \sim 1 / H$ the Hubble length. The energy density inside the domain wall is of $\mathcal{O}\left(m_{a}^{2} v_{\mathrm{PQ}}^{2}\right)$. The typical thickness of the domain wall is given by $m_{a}(T)^{-1}[54,55]$, where

$$
m_{a}(T)^{2} \simeq \frac{\sqrt{2} \lambda}{m !} \frac{M_{\mathrm{Pl}}^{3}}{v_{\mathrm{PQ}}}\left[\frac{2(n-3)(n !)^{2}}{n(n-1)^{2} \lambda_{s}^{2}}\right]^{\frac{m}{2(n-1)}}\left(\frac{H}{M_{\mathrm{Pl}}}\right)^{\frac{m}{n-1}}
$$

\footnotetext{
${ }^{7}$ Here, $P$ does not necessarily follow the minimum of the potential, although such a behavior does not affect the following argument.

${ }^{8} S$ and $P$ mix through the $S^{m} P$ term; however, the mixing is not large enough for $S$ to be thermalized.

${ }^{9} \mathrm{We}$ assume $\lambda_{p}$ and $y_{\mathrm{KSVZ}}$ are of $\mathcal{O}(1)$.
}

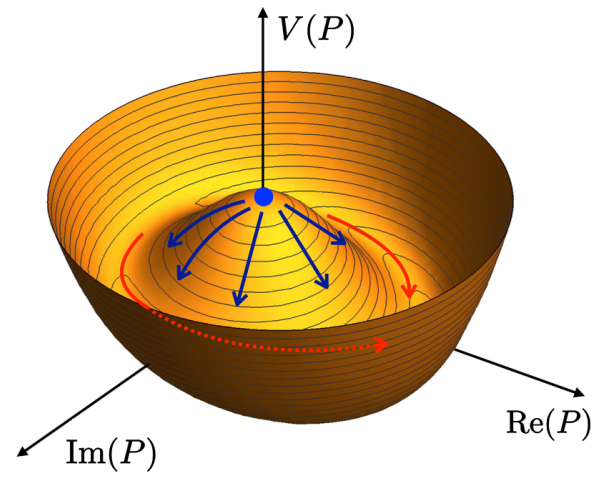

FIG. 1. A schematic picture of the axion evolution when $P$ obtains the VEV. The tilted Mexican hat corresponds to the scalar potential of the PQ field on a complex plane of $P$. In each Hubble patch, the axion rolls down from the hilltop of the potential in a random direction. The axion settles to a unique field value due to the bias term made by the large field value of $S$.

is induced by the $P-S$ mixing. The typical radius of the cosmic string is given by $v_{\mathrm{PQ}}^{-1}$ for $\lambda_{p}=\mathcal{O}(1)$ [56], with the energy density inside the cosmic string of $v_{\mathrm{PQ}}^{4}$. From these quantities, we obtain

$$
\begin{aligned}
\sigma_{\mathrm{w}} & \simeq 8 m_{a}(T) v_{\mathrm{PQ}}^{2}, \\
T_{\mathrm{s}} & \simeq 2 \pi v_{\mathrm{PQ}}^{2} \ln \left(v_{\mathrm{PQ}} / H(T)\right) .
\end{aligned}
$$

By plugging Eq. (39) into Eq. (37), the condition Eq. (37) is reduced to

$$
m_{a}(T)>\frac{\pi}{4} H(T) \ln \left(\frac{v_{\mathrm{PQ}}}{H(T)}\right) .
$$

The string-wall network immediately shrinks and collapses when this condition is satisfied for some temperature after $P$ obtains the VEV.

It should be noted that the phase component of $S$ also participates in the scalar potential induced by the $P-S$ mixing. Unlike the axion, however, it does not move during the above process. Thus, we can neglect the motion of the phase component of $S$ in the above argument. To see this behavior, let us decompose the $S$ field by

$$
S=\frac{\chi}{\sqrt{2}} \exp \left(i \frac{b}{\chi}\right)
$$

where $b$ is the phase component of $S$. The field value of the radial component $\chi$ slowly decreases according to the scaling solution [see Eqs. (24) and (31)]. The scalar potential of $a$ and $b$ induced by the $P-S$ mixing term is given by ${ }^{10}$

\footnotetext{
${ }^{10}$ Here, we redefine the origin of $b$ so that the minimum of the potential is at $a=b=0$.
} 

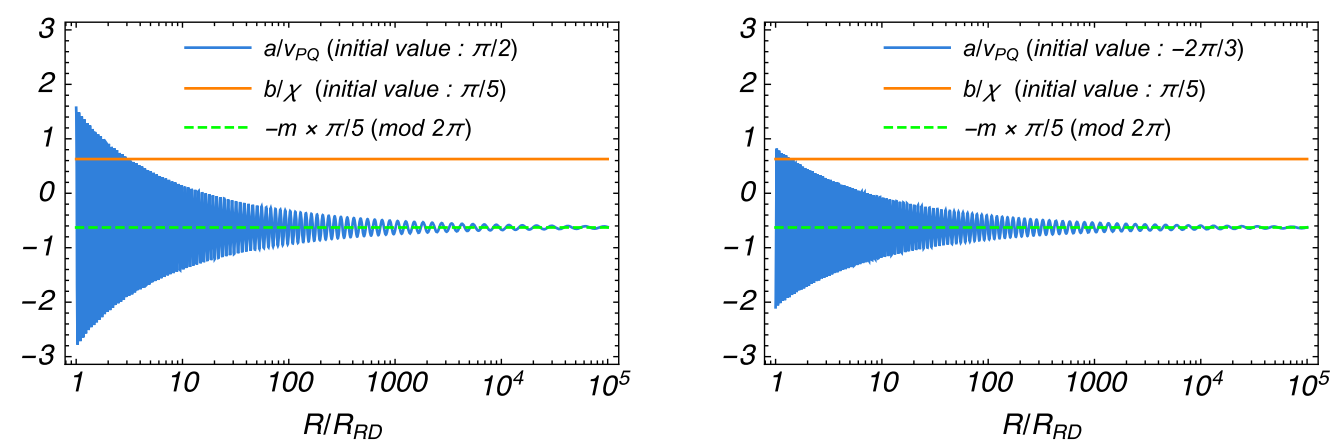

FIG. 2. The behaviors of $a$ and $b$ during the RD era at a benchmark point $n=6, m=11, \lambda_{p}=c_{s}=c_{p}=1, \lambda_{s}=10^{-4}, \lambda=10^{-6}$, and $v_{\mathrm{PQ}}=10^{12} \mathrm{GeV}$. The initial conditions of the phase components are $a / v_{\mathrm{PQ}}=\pi / 2$ (left) and $a / v_{\mathrm{PQ}}=-3 \pi / 2$, while $b / \chi=\pi / 5$ in both panels. In both cases, $a / v_{\mathrm{PQ}}$ settles around $-m \pi / 5(\bmod 2 \pi)=-\pi / 5$, while $b / \chi$ is a constant in time.

$V(a, b)=2 \frac{\lambda}{m ! M_{\mathrm{Pl}}^{m-3}}\left(\frac{\chi}{\sqrt{2}}\right)^{m} \frac{v_{\mathrm{PQ}}}{\sqrt{2}}\left[1-\cos \left(\frac{a}{v_{\mathrm{PQ}}}+m \frac{b}{\chi}\right)\right]$.

This scalar potential implies that the axion oscillates much faster than $b$ since $\chi \gg v_{\mathrm{PQ}}$ at $T \sim v_{\mathrm{PQ}}$. Thus, $a$ oscillates around $-m b v_{\mathrm{PQ}} / \chi(\bmod 2 \pi)$, while $b$ does not feel the force from the potential since it is averaged out by the oscillation of $a .^{11}$

In Fig. 2, we show the behaviors of $a$ and $b$ during the $\mathrm{RD}$ era at a benchmark point, $n=6, m=11, \lambda_{p}=c_{s}=$ $c_{p}=1, \lambda_{s}=10^{-4}, \lambda=10^{-6}$, and $v_{\mathrm{PQ}}=10^{12} \mathrm{GeV}$ (see Sec. IVE). We start the analysis just after $P$ obtains the VEV at $T \sim 10^{12} \mathrm{GeV}$. $\chi$ is assumed to follow the scaling solution in Eq. (31). As the initial conditions of the phase components, we take $a / v_{\mathrm{PQ}}=\pi / 2$ (left) and $a / v_{\mathrm{PQ}}=$ $-2 \pi / 3$ (right), while $b / \chi=\pi / 5$, respectively. The initial velocities are taken to be zero. The figure shows that $a / v_{\mathrm{PQ}}$ settles around $-m v_{\mathrm{PQ}} b / \chi(\bmod 2 \pi)$. The figure also shows that $b / \chi$ is almost constant in time as expected. The oscillation period of $a / v_{\mathrm{PQ}}$ becomes longer at the later time, as $\chi$ decreases according to the scaling solution. In this way, the random field value of $a$ in each Hubble patch settles in the vicinity of the uniform field value $-m b v_{\mathrm{PQ}} / \chi(\bmod 2 \pi)$.

\section{The axion dynamics after the onset of $S$ oscillation}

As we have mentioned earlier, $S$ starts to oscillate around its origin when the Hubble parameter becomes smaller than $m_{s}$. In this subsection, we consider the dynamics of the axion after the onset of the $S$ oscillation.

Because the axion potential is induced by the mixing term, the sign of the axion potential also flips when $S$ oscillates (see Fig. 3). If the axion mass exceeds the Hubble parameter at that time, the axion falls from the top of the

\footnotetext{
${ }^{11}$ More precisely, $b$ settles to $b_{i}-m a_{i} v_{\mathrm{PQ}} / \chi+\mathcal{O}\left(b_{i} m^{2} v_{\mathrm{PQ}}^{2} / \chi^{2}\right)$.
}

flipped potential. If this happens, the axion field value in each Hubble patch is again randomized and can no longer be uniform in our Universe. Such a behavior brings back the domain wall problem.

To avoid this situation, we require that the Hubble friction on the axion is effective when $S$ starts to oscillate. This condition can be expressed in the form

$$
m_{a}(T)<3 H=m_{s}
$$

where we estimate the onset of the $S$ oscillation by $3 H=m_{s} \cdot{ }^{12}$

As we have seen in Eq. (40), the axion mass should exceed the Hubble parameter when $P$ obtains the VEV. On the other hand, the axion mass needs to be smaller than the Hubble friction when $3 H=m_{s}$. These two requirements lead to the condition that the axion mass in Eq. (38) $m_{a} \propto$ $H^{\frac{m}{2(n-1)}}$ must decrease faster than the Hubble parameter. Thus, the above condition is satisfied for

$$
m \geq 2 n-1 \text {. }
$$

To this point, we have ignored the backreaction to $S$ from $P$. As we have seen, $\left\langle S_{I}\right\rangle \gg\left\langle P_{I}\right\rangle$ during inflation. Thus, the backreaction from $P$ through the mixing term is negligible during inflation. As the field value of $P$ decreases much faster than that of $S$, the backreaction is also negligible in the inflaton oscillation era and the RD era until $P$ obtains the VEV.

After $P$ obtains the $\mathrm{VEV}\langle P\rangle \simeq v_{\mathrm{PQ}}$, the backreaction could modify the behavior of $S$ in Eq. (31) if ${ }^{13}$

\footnotetext{
${ }^{12}$ Below the temperature of $m_{a}(T) \simeq 3 H$, the field value of $a$ is frozen to a different field value in each Hubble patch. The difference of the frozen filed value, however, does not cause the domain wall problem as long as all the difference is smaller than $2 \pi f_{a}$.

${ }^{13}$ Here, we use the lower limit on $m$ in Eq. (44) since the backreaction is weaker for a larger $m$.
} 


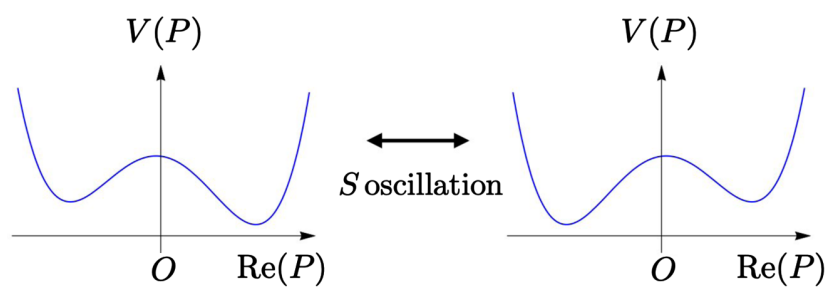

FIG. 3. A schematic picture of the behavior of the scalar potential of the PQ field. Here, we show the sectional view of Fig. 1 on the $V(P)-\operatorname{Re}(P)$ plane. As the spectator PQ field oscillates, the sign of the bias in the axion potential flips.

$$
\begin{aligned}
\frac{\lambda_{s}^{2}}{(n !)^{2} M_{\mathrm{Pl}}^{2 n-4}}|S|^{2 n} & \sim \frac{\lambda}{(2 n-1) ! M_{\mathrm{Pl}}^{2 n-4}}|S|^{2 n-1} v_{\mathrm{PQ}} \\
\therefore S & \sim \frac{(n !)^{2} \lambda}{(2 n-1) ! \lambda_{s}^{2}} v_{\mathrm{PQ}} .
\end{aligned}
$$

However, such a situation can be avoided if the mass of $S$ is larger so that $S$ starts oscillation before Eq. (45) is satisfied. Thus, so long as

$$
\lambda v_{\mathrm{PQ}} M_{\mathrm{Pl}}\left(\frac{(n !)^{2} \lambda}{m ! \lambda_{s}^{2}} \frac{v_{\mathrm{PQ}}}{M_{\mathrm{Pl}}}\right)^{2 n-3}<m_{s}^{2}
$$

we can safely neglect the backreaction of $P$ to the dynamics of $S$.

\section{Isocurvature perturbations of the axion}

Because $P$ obtains the VEV during the RD era, it seems that the axion does not suffer from the isocurvature problem. However, the phase component $b$ of $S$ has a flat potential when $S$ takes a large field value, and it obtains quantum fluctuation during inflation. In the presence of the mixing term, the fluctuation of the phase component of $S$ is imprinted in the axion, which leads to the isocurvature perturbations of the axion dark matter.

During inflation, the fluctuation of $b$ is given by [57-61]

$$
\delta b_{I} \simeq \frac{H_{I}}{2 \pi},
$$

where $\left\langle\chi_{I}\right\rangle=\sqrt{2}\left\langle S_{I}\right\rangle .{ }^{14}$ After inflation, $S$ follows the scaling solution which is along the straight line passing through $S=0$ in the complex plane of $S$. Thus, the fluctuation of $\delta b$ decreases as

$$
\delta b \simeq \frac{\chi}{\left\langle\chi_{I}\right\rangle} \times \delta b_{I} \simeq \frac{H_{I}}{2 \pi} \frac{\chi}{\left\langle\chi_{I}\right\rangle} .
$$

\footnotetext{
${ }^{14}$ Since $P$ also takes a large field value, $a$ also fluctuates during inflation. However, the axion eventually settles around $-m v_{\mathrm{PQ}} / \chi b$ regardless of its initial value, as we have seen in Sec. IV B. Thus, the fluctuation of $a$ does not affect the following arguments.
}

Once $P$ obtains the VEV, the $P-S$ mixing term leads to the potential of $a$ and $b$ in Eq. (42). Then, as we discussed in Sec. IV B, $a / v_{\mathrm{PQ}}$ settles around $-m \times b / \chi(\bmod 2 \pi)$ while $b / \chi$ does not move. As a result, the fluctuation of $b$ is imprinted in $a$ as ${ }^{15}$

$$
\frac{\delta a}{f_{a}} \simeq m \frac{v_{\mathrm{PQ}}}{\chi} \frac{\delta b}{f_{a}} \simeq \frac{m N_{\mathrm{DW}} H_{I}}{2 \pi\left\langle\chi_{I}\right\rangle} .
$$

Below the QCD scale, the axion starts coherent oscillation, and the axion fluctuation results in the uncorrelated isocurvature perturbations. The power spectrum of the isocurvature perturbations is given by

$$
\mathcal{P}_{I} \simeq 8\left(\frac{\delta a}{f_{a}}\right)^{2}\left(\frac{f_{a}}{10^{12} \mathrm{GeV}}\right)^{1.19}\left(\frac{\Omega_{a} h^{2}}{0.1}\right)
$$

where we assume that the observed dark matter density is dominated by the axion. From the CMB observations, the uncorrelated isocurvature perturbations of cold dark matter are constrained by [31]

$$
\beta_{\text {iso }}=\frac{\mathcal{P}_{I}}{\mathcal{P}_{\zeta}+\mathcal{P}_{I}} \leq 0.038
$$

where $\mathcal{P}_{\zeta} \simeq 2 \times 10^{-9}$ denotes the power spectrum of the curvature perturbations. By combining Eqs. (13), (49), and (50), we find the upper limit on $H_{I}$ is given by

$$
\begin{aligned}
H_{I} \lesssim & M_{\mathrm{Pl}} \times\left(8 \times 10^{-10} \frac{1}{m^{2} N_{\mathrm{DW}}^{2}}\left(\frac{10^{12} \mathrm{GeV}}{f_{a}}\right)^{1.19}\right. \\
& \left.\times\left(\frac{0.1}{\Omega_{a} h^{2}}\right)\right)^{\frac{n-1}{2(n-2)}}\left(\sqrt{\frac{c_{s}}{n}} \frac{n !}{\lambda_{s}}\right)^{\frac{1}{n-2}} .
\end{aligned}
$$

\section{E. Viable parameter region}

Let us summarize the constraints on the model parameters. In Fig. 4, we show the necessary conditions on the $\left(m_{s}, \lambda\right)$ plane for $N_{\mathrm{DM}}=1$ (the minimal $\mathrm{KSVZ}$ axion model) and for $N_{\mathrm{DM}}=6$ [the Dine-Fischler-SrednickiZhitnitsky (DFSZ) axion model $[7,8]] .{ }^{16}$ We take $n=6$, $m=11, \lambda_{p}=1, c_{s}=1$, and $c_{p}=1$ as a benchmark point. These conditions are given in the form of inequalities (40), (43), and (46). The blue shaded region is excluded where the condition in Eq. (40) is not satisfied after $P$ obtains the $\mathrm{VEV}$, and hence, the randomness of the axion direction is not resolved. The orange shaded region is excluded where

\footnotetext{
${ }^{15}$ Only the fluctuation modes longer than the Hubble length at the QCD temperature are relevant for the isocurvature perturbations of the axion dark matter, which are the superhorizon mode when $a$ settles around $-m v_{\mathrm{PQ}} / \chi b$.

${ }^{16}$ Here, we assume that the two Higgs doublets in the DFSZ model couple to the PQ field via $P^{2} H_{1} H_{2}$.
} 

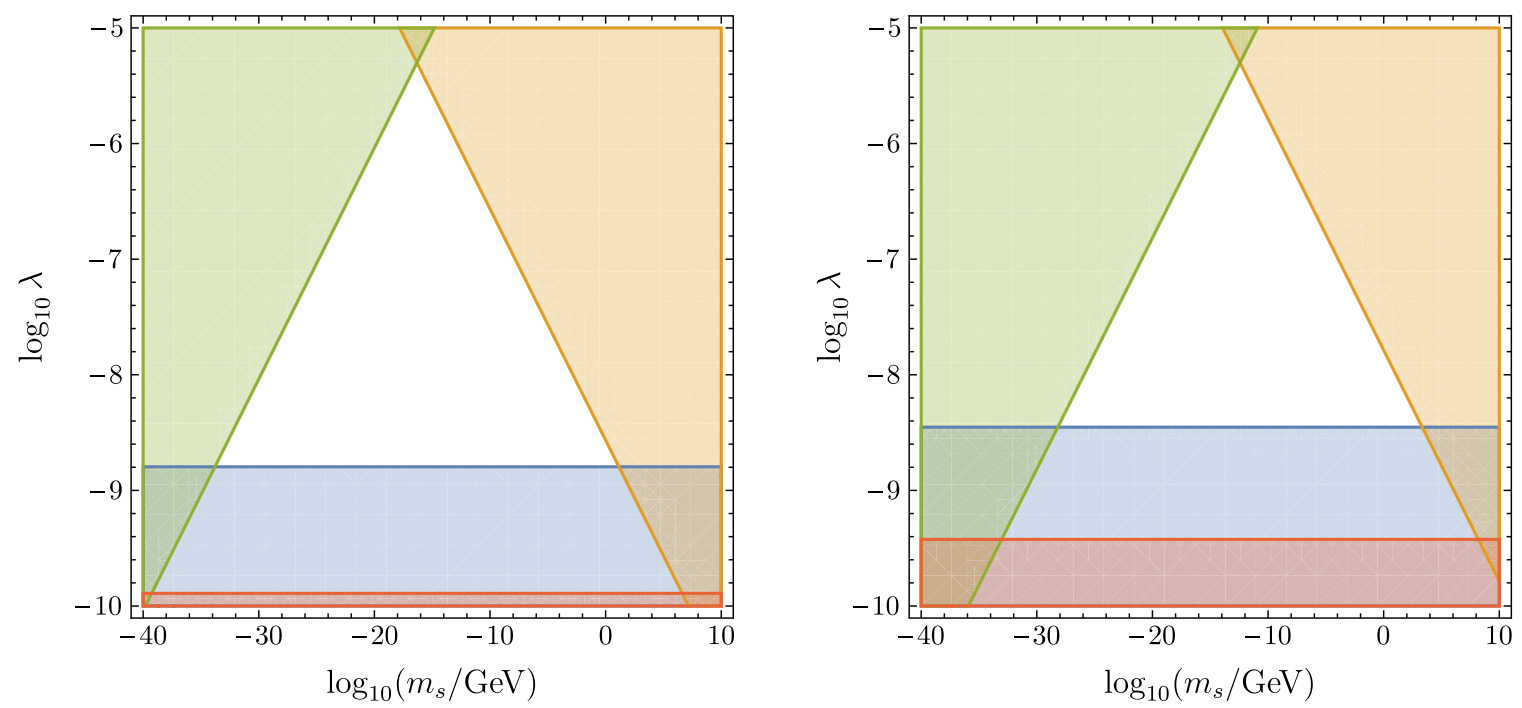

FIG. 4. Various constraints on the model parameters. The blue, orange, and green shaded areas are excluded by the conditions in Eqs. (40), (43), and (46). The red one is excluded by a conservative condition $m_{a}\left(T \sim v_{\mathrm{PQ}}\right) \gtrsim 3 H\left(T \sim v_{\mathrm{PQ}}\right)$. As a benchmark, we set $n=6, m=11$, and $\lambda_{p}=c_{p}=c_{s}=1$, and $\lambda_{s}=10^{-4}$. (Left) $N_{\mathrm{DW}}=1$; (right) $N_{\mathrm{DW}}=6$.

the condition in Eq. (43) is not satisfied at $3 H \simeq m_{s}$, and hence, the axion is randomized as $S$ oscillates. The green shaded region is excluded where the condition in Eq. (46) is not satisfied, where the backreaction from $P$ to $S$ becomes sizable. The red shaded region shows a more conservative constraint $m_{a}\left(T \sim v_{\mathrm{PQ}}\right) \gtrsim 3 H\left(T \sim v_{\mathrm{PQ}}\right)$, which is weaker than that in Eq. (40). This weaker constraint is good enough if no cosmic strings are formed when $P$ obtains the VEV at $T \sim v_{\mathrm{PQ}}$. The figure shows that all conditions are satisfied for a wide range of $m_{s}$. For the minimal KSVZ model, i.e., $N_{\mathrm{DW}}=1, m_{s} \lesssim 1 \mathrm{GeV}$, and for the DFSZ model, $m_{s} \lesssim 10^{2} \mathrm{GeV}$.

For the benchmark scenario, $n=6, m=11$, the constraint on the isocurvature perturbations leads to

$$
H_{I} \lesssim 10^{12} \mathrm{GeV} \times \frac{1}{N_{\mathrm{DW}}^{5 / 4}}\left(\frac{\sqrt{c_{s}}}{\lambda_{s}}\right)^{1 / 4}
$$

Thus, we find that the present mechanism allows $H_{I} \sim$ $10^{13} \mathrm{GeV}$ for $\lambda_{s} \sim 10^{-6} .^{17}$

As a result, we find that the present mechanism makes the axion model with $N_{\mathrm{DM}} \neq 1$ compatible with the inflation model in which a large Hubble parameter is rather large, i.e., $H_{I} \gg 10^{7-8} \mathrm{GeV}$. Therefore, this mechanism increases the freedom of choice of the combinations of the axion models and inflation models.

\footnotetext{
${ }^{17}$ For $\lambda_{s} \sim 10^{-6}$, the expectation value of $S$ during inflation slightly exceeds $M_{\mathrm{Pl}}$, which requires a small $c_{s}$ to avoid too much potential energy of $S$ during inflation.
}

\section{SUPERSYMMETRIC REALIZATION}

As we have seen in the previous section, the model requires the higher-dimensional interaction terms with specific exponents. Such scalar potentials are not easily justified in nonsupersymmetric theories. In this section, we briefly discuss a supersymmetric realization of the mechanism to make the scenario more viable, where the choices of the mass parameters in the model are technically natural. A detailed analysis of the supersymmetric extension will be given elsewhere.

For example, a model with $n=6$ and $m=11$ can be easily realized by assuming a superpotential,

$$
\begin{aligned}
W= & X\left(P \bar{P}-v_{\mathrm{PQ}}^{2}\right)+\frac{1}{M_{\mathrm{Pl}}^{4}} Y S^{6}+Z\left(S^{2} P+\frac{1}{M_{\mathrm{Pl}}^{6}} S^{9}\right) \\
& +\frac{1}{M_{\mathrm{Pl}}^{6}} X \bar{P} S^{7} .
\end{aligned}
$$

Here, $P$ and $S$ are the chiral superfields corresponding to the (spectator) PQ fields, while $X, Y$, and $Z$ are chiral superfields whose $F$ components lead to the scalar potential in Eq. (8). ${ }^{18}$ We omit the coupling constants for brevity. The PQ charge assignment is given in Table I. This model justifies why the potential term with a lower dimension than $|S|^{2 n}$ is absent. The mass term of the scalar component of $S$ is generated by the supersymmetry breaking effects. It should be noted that the unwanted superpotential terms

\footnotetext{
${ }^{18}$ In this realization, the $P-S$ mixing term in the scalar potential is given by $|S|^{4} S^{7} P^{*}+$ H.c. Accordingly, the cosine potential of the phase components in Eq. (42) is modified to $\cos \left(a / v_{\mathrm{PQ}}+7 b / \chi\right)$.
} 
TABLE I. Charge assignments of the supersymmetric model. In addition to the PQ symmetry, we show the $R$ symmetry.

\begin{tabular}{lcccccccc}
\hline \hline & $X$ & $Y$ & $Z$ & $S$ & $P$ & $\bar{P}$ & $Q_{L} \bar{Q}_{R}$ & $\psi_{s} \bar{\psi}_{s}$ \\
\hline$U(1)_{\mathrm{PQ}}$ & 0 & -6 & -9 & 1 & 7 & -7 & -7 & -1 \\
$R$ & 2 & 2 & 2 & 0 & 0 & 0 & 2 & 2 \\
\hline \hline
\end{tabular}

such as $Y P^{i} S^{j}$ with $i>1(i+j=6)$ are suppressed by PQ and $R$ symmetry.

The last term of Eq. (54) induces

$$
V=\frac{1}{M_{\mathrm{Pl}}^{6}}\left(P \bar{P}-v_{\mathrm{PQ}}^{2}\right) \bar{P}^{*} S^{7}+\text { H.c. }
$$

When $T \gtrsim v_{\mathrm{PQ}}, P$ and $\bar{P}$ are settled to the origin due to thermal mass terms; thus, this term does not affect the dynamics of $S$ and $P$. In addition, after $\langle P \bar{P}\rangle$ settles to $v_{\mathrm{PQ}}^{2}$, this term vanishes. Therefore, this term does not have an influence on the dynamics all through the epoch of interest.

We briefly comment on the effects of higher-dimensional terms in the Kähler potential. For example, a higherdimensional operator

$$
K=\frac{1}{M_{\mathrm{Pl}}^{2}}|X|^{2}|S|^{2}
$$

induces a scalar potential

$$
V=\frac{\left|P \bar{P}-v_{\mathrm{PQ}}^{2}\right|^{2}}{1+|S|^{2} / M_{\mathrm{Pl}}^{2}}
$$

This term leads to an additional effective mass of $S$ for a given $\langle P \bar{P}\rangle$. During inflation, this term is smaller than the Hubble-induced mass term [see Eqs. (33) and (34)]. After inflation, $\langle P \bar{P}\rangle$ immediately vanishes, and the mass term in Eq. (57) leads to a mass of $\mathcal{O}\left(v_{\mathrm{PQ}}^{2} / M_{\mathrm{Pl}}\right)$, which is smaller than the Hubble constant until $T \sim v_{\mathrm{PQ}}$. After $\langle P \bar{P}\rangle$ settles to $v_{\mathrm{PQ}}^{2}$, the induced mass vanishes. ${ }^{19}$ Therefore, we find that the induced mass does not affect the dynamics of the scalar fields.

In addition to the higher-dimensional term in Eq. (56), there are terms

$$
K=\frac{1}{M_{\mathrm{Pl}}^{2}}\left(|Y|^{2}|S|^{2}+|Z|^{2}|S|^{2}+|S|^{4}\right)+\cdots
$$

These terms just induce additional terms with higher dimension than those in Eq. (8), and hence, they do not

\footnotetext{
${ }^{19}$ In the presence of the supersymmetry breaking effects, the VEV of $P \bar{P}$ is slightly shifted from $v_{P Q}^{2}$, and hence, the effective mass in Eq. (57) does not vanish completely. However, we can set this mass term small enough not to affect the dynamics.
}

affect the scaling behavior of $S .{ }^{20}$ In the case of $|S|^{4}$, we redefine $S$ so that it has a canonical kinetic term. With the redefinition, the effects appear as the additional terms with higher dimension than those in Eq. (8). The same is true for the higher-dimensional Kähler term of $|S|^{2 k}(k>1)$.

Supersymmetric extension is also advantageous to explain the interactions between the PQ fields and the inflaton in Eq. (9). In fact, a Kähler potential

$$
K=\frac{c_{p}}{3 M_{\mathrm{Pl}}^{2}}|I|^{2}|P|^{2}-\frac{c_{s}}{3 M_{\mathrm{Pl}}^{2}}|I|^{2}|S|^{2}
$$

explains the interactions between the PQ fields and the inflaton. Here, $I$ denotes the chiral superfield of the inflaton $\phi$ whose $F$ component provides the inflaton potential, i.e., $V(\phi)=\left|F_{I}\right|^{2}$.

In addition to Eq. (59), we can write higher order Kähler terms such as

$$
K=|I|^{2}\left(\frac{|S|^{4}}{M_{\mathrm{Pl}}^{4}}+\frac{|S|^{6}}{M_{\mathrm{Pl}}^{6}}+\cdots\right) .
$$

In the case of $\left\langle S_{I}\right\rangle \sim M_{\mathrm{Pl}}$, these terms slightly modify the expectation value of $S$ during inflation. However, after inflation, $\langle S\rangle$ starts to decrease, then the higher order contributions become negligible; therefore, they do not affect the dynamics.

Finally, here are some comments on the origin of $m_{S}$. In the supersymmetric model, $m_{S}$ is generated by some mediation mechanism of supersymmetry breaking effects. From the null results of the supersymmetric particle searches at the LHC, the lower limit on the sfermion masses are $\mathcal{O}(1) \mathrm{TeV}[62,63]$. The required mass of the scalar component of $S$ is, however, at most $100 \mathrm{GeV}$. Thus, $m_{S} \lesssim 100 \mathrm{GeV}$ requires fine-tuning in the gravity mediation mechanism since it predicts $m_{S}$ close to the sfermion masses.

In gauge mediation scenarios, on the other hand, $m_{S}$ can be as light as the gravitino mass $m_{3 / 2}$, while the sfermion masses can be much larger. In this way, we can achieve a small value of $m_{S}$ without serious fine-tuning. The detailed study of the viable range of small $m_{S}$ and $m_{3 / 2}$ will be discussed elsewhere.

\section{CONCLUSIONS}

The domain wall problem and the isocurvature problem restrict possible combinations of axion models and inflation models. In this paper, we considered a new mechanism which solves those problems by introducing the spectator PQ field which obtains a large field value before the PQ field obtains the VEV. The mechanism makes the axion

\footnotetext{
${ }^{20}$ We assume that $X, Y, Z$ obtain the positive Hubble mass terms; hence, they settle at the origin.
} 
model with a nontrivial domain wall number compatible with the inflation model with a large Hubble parameter $H_{I} \gg 10^{7-8} \mathrm{GeV}$. The mechanism is also free from the isocurvature problem. It should be emphasized that this mechanism can be added to any conventional axion model. Thus, this mechanism increases the freedom of choice of combinations of axion models and inflation models.

We also found that the present mechanism can be consistent with a large Hubble parameter during inflation of $H_{I} \sim 10^{13} \mathrm{GeV}$. Thus, the scenario can be tested by combining future axion search experiments and the searches for the primordial $B$-mode polarization in the CMB.

We also note that the coherent oscillation of the spectator PQ field can play the role of dark matter when it is very light, as we discussed in Sec. III C. ${ }^{21}$ As the initial amplitude of the coherent oscillation is dynamically determined, this model realizes a very light scalar dark matter without fine-tuning of the initial condition in an alternative way to the axionlike ultralight dark matter in $[64,65]$. Such a very light dark matter can be tested via astronomical ephemeris [66].

\section{ACKNOWLEDGMENTS}

The authors thank M. Kawasaki for an important comment on the isocurvature problem. This work is supported in part by JSPS KAKENHI Grants No. 15H05889, No. 16H03991, No. $18 \mathrm{H} 05542$ (M. I.), No. 16H02176 (T. T. Y), No. 17 H02878 (M. I. and T. T. Y.); World Premier International Research Center Initiative (WPI Initiative),

\footnotetext{
${ }^{21}$ In this case, we do not need to introduce the light fermions in Eq. (32).
}

MEXT, Japan (M. I. and T. T. Y). T. T. Y. is supported in part by the China Grant for Talent Scientific Start-Up Project. T. T. Y. thanks Hamamatsu Photonics. The work of S. K. is supported by the Advanced Leading Graduate Course for Photon Science.

\section{APPENDIX: ENERGY DENSITY OF THE SPECTATOR PQ FIELD}

In this appendix, we discuss the energy density of the spectator PQ field $S$. When $S$ follows the scaling solution in Eqs. (13), (24), and (31), the potential energy density of $S$ is of $\mathcal{O}\left(H^{2}|S|^{2}\right)$. Thus, it is subdominant compared with the dominant energy density of $\mathcal{O}\left(H^{2} M_{\mathrm{Pl}}^{2}\right)$ as long as $\langle S\rangle \ll M_{\mathrm{Pl}}$.

Once $S$ starts coherent oscillation around its origin, $S$ behaves as massive matter with an energy density

$$
\rho_{S}=m_{s}^{2}|S|^{2}
$$

The radiation density at that time is $m_{s}^{2} M_{\mathrm{Pl}}^{2} / 3$ where we have used $H \simeq m_{s} / 3$. Thus, again, the energy density of $S$ is subdominant since $S \ll M_{\mathrm{Pl}}$ at the onset of the coherent oscillation.

As we considered in Sec. II, $S$ immediately decays into the massless fermions which behave as radiation. Thus, the energy density of $S$ does not causes any cosmological problems. As the energy density of $S$ is subdominant, the energy density of the massless fermions is also subdominant. Furthermore, the relative entropy of the massless fermions is diluted when all the entropy in the thermal bath goes into the particles in the standard cosmology (i.e., the photons and the neutrinos). Thus, the contributions of the massless fermions to dark radiation are also negligible.
[1] R. D. Peccei and H. R. Quinn, Phys. Rev. Lett. 38, 1440 (1977).

[2] R. D. Peccei and H. R. Quinn, Phys. Rev. D 16, 1791 (1977).

[3] S. Weinberg, Phys. Rev. Lett. 40, 223 (1978).

[4] F. Wilczek, Phys. Rev. Lett. 40, 279 (1978).

[5] J. E. Kim, Phys. Rev. Lett. 43, 103 (1979).

[6] M. A. Shifman, A. I. Vainshtein, and V. I. Zakharov, Nucl. Phys. B166, 493 (1980).

[7] A. R. Zhitnitsky, Yad. Fiz. 31, 497 (1980) [Sov. J. Nucl. Phys. 31, 260 (1980)].

[8] M. Dine, W. Fischler, and M. Srednicki, Phys. Lett. 104B, 199 (1981).

[9] J. Preskill, S. P. Trivedi, F. Wilczek, and M. B. Wise, Nucl. Phys. B363, 207 (1991).

[10] L. F. Abbott and P. Sikivie, Phys. Lett. 120B, 133 (1983).
[11] J. Preskill, M. B. Wise, and F. Wilczek, Phys. Lett. 120B, 127 (1983).

[12] M. Dine and W. Fischler, Phys. Lett. 120B, 137 (1983).

[13] M. Kawasaki and K. Nakayama, Annu. Rev. Nucl. Part. Sci. 63, 69 (2013).

[14] M. Kawasaki, T. T. Yanagida, and K. Yoshino, J. Cosmol. Astropart. Phys. 11 (2013) 030.

[15] A. Vilenkin and A. E. Everett, Phys. Rev. Lett. 48, 1867 (1982).

[16] T. Hiramatsu, M. Kawasaki, K. Saikawa, and T. Sekiguchi, Phys. Rev. D 85, 105020 (2012); 86, 089902(E) (2012).

[17] M. Kawasaki and E. Sonomoto, Phys. Rev. D 97, 083507 (2018).

[18] P. W. Graham, I. G. Irastorza, S. K. Lamoreaux, A. Lindner, and K. A. van Bibber, Annu. Rev. Nucl. Part. Sci. 65, 485 (2015). 
[19] S. M. Barr and J. E. Kim, Phys. Rev. Lett. 113, 241301 (2014).

[20] M. Reig, J. High Energy Phys. 08 (2019) 167.

[21] A. Caputo and M. Reig, Phys. Rev. D 100, 063530 (2019).

[22] D. Stojkovic, K. Freese, and G. D. Starkman, Phys. Rev. D 72, 045012 (2005).

[23] T. Hiramatsu, M. Kawasaki, K. Saikawa, and T. Sekiguchi, J. Cosmol. Astropart. Phys. 01 (2013) 001.

[24] M. Kawasaki, K. Saikawa, and T. Sekiguchi, Phys. Rev. D 91, 065014 (2015).

[25] M. Axenides, R. H. Brandenberger, and M. S. Turner, Phys. Lett. 126B, 178 (1983).

[26] D. Seckel and M. S. Turner, Phys. Rev. D 32, 3178 (1985).

[27] A. D. Linde, Phys. Lett. 158B, 375 (1985).

[28] A. D. Linde and D. H. Lyth, Phys. Lett. B 246, 353 (1990).

[29] M. S. Turner and F. Wilczek, Phys. Rev. Lett. 66, 5 (1991).

[30] D. H. Lyth, Phys. Rev. D 45, 3394 (1992).

[31] Y. Akrami et al. (Planck Collaboration), Astrophys. Space Sci. 364, 69 (2019); arXiv:1807.06211.

[32] M. Kawasaki, E. Sonomoto, and T. T. Yanagida, Phys. Lett. B 782, 181 (2018).

[33] N. Du et al. (ADMX Collaboration), Phys. Rev. Lett. 120, 151301 (2018).

[34] A. Caldwell, G. Dvali, B. Majorovits, A. Millar, G. Raffelt, J. Redondo, O. Reimann, F. Simon, and F. Steffen (MADMAX Working Group), Phys. Rev. Lett. 118, 091801 (2017).

[35] J. W. Foster, N. L. Rodd, and B. R. Safdi, Phys. Rev. D 97, 123006 (2018).

[36] D. Alesini, D. Babusci, D. Di Gioacchino, C. Gatti, G. Lamanna, and C. Ligi, arXiv:1707.06010.

[37] K. N. Abazajian et al. (CMB-S4 Collaboration), arXiv: 1610.02743 .

[38] A. D. Linde, Phys. Lett. B 259, 38 (1991).

[39] K. Harigaya, M. Ibe, M. Kawasaki, and T. T. Yanagida, J. Cosmol. Astropart. Phys. 11 (2015) 003.

[40] F. Takahashi and M. Yamada, J. Cosmol. Astropart. Phys. 10 (2015) 010.

[41] M. Kawasaki, F. Takahashi, and M. Yamada, Phys. Lett. B 753, 677 (2016).

[42] J. Kearney, N. Orlofsky, and A. Pierce, Phys. Rev. D 93, 095026 (2016).

[43] S.-Y. Ho, K. Saikawa, and F. Takahashi, J. Cosmol. Astropart. Phys. 10 (2018) 042.
[44] E. Witten, Phys. Lett. 149B, 351 (1984).

[45] R. Kallosh, A. D. Linde, D. A. Linde, and L. Susskind, Phys. Rev. D 52, 912 (1995).

[46] P. Svrcek and E. Witten, J. High Energy Phys. 06 (2006) 051.

[47] M. Kawasaki, F. Takahashi, and M. Yamada, J. High Energy Phys. 01 (2018) 053.

[48] R. T. Co, E. Gonzalez, and K. Harigaya, J. High Energy Phys. 05 (2019) 162.

[49] O. Wantz and E. P. S. Shellard, Nucl. Phys. B829, 110 (2010).

[50] G. Grilli di Cortona, E. Hardy, J. Pardo Vega, and G. Villadoro, J. High Energy Phys. 01 (2016) 034.

[51] K. J. Bae, J.-H. Huh, and J. E. Kim, J. Cosmol. Astropart. Phys. 09 (2008) 005.

[52] A. R. Liddle and R. J. Scherrer, Phys. Rev. D 59, 023509 (1998).

[53] Y. Ema, K. Nakayama, and M. Takimoto, J. Cosmol. Astropart. Phys. 02 (2016) 067.

[54] A. Vilenkin, Phys. Rev. Lett. 72, 3137 (1994).

[55] A. D. Linde, Phys. Lett. B 327, 208 (1994).

[56] A. Vilenkin and E. P.S. Shellard, Cosmic Strings and Other Topological Defects (Cambridge University Press, Cambridge, England, 2000).

[57] V. F. Mukhanov and G. V. Chibisov, Pis'ma Zh. Eksp. Teor. Fiz. 33, 549 (1981) [JETP Lett. 33, 532 (1981)]; http://www .jetpletters.ac.ru/ps/1510/article_23079.shtml.

[58] S. W. Hawking, Phys. Lett. 115B, 295 (1982).

[59] A. A. Starobinsky, Phys. Lett. 117B, 175 (1982).

[60] A. H. Guth and S. Y. Pi, Phys. Rev. Lett. 49, 1110 (1982).

[61] J. M. Bardeen, P. J. Steinhardt, and M. S. Turner, Phys. Rev. D 28, 679 (1983).

[62] CERN Technical Report No. ATL-PHYS-PUB-2019-044, 2019.

[63] A. M. Sirunyan et al. (CMS Collaboration), J. High Energy Phys. 10 (2019) 244.

[64] W. Hu, R. Barkana, and A. Gruzinov, Phys. Rev. Lett. 85, 1158 (2000).

[65] L. Hui, J. P. Ostriker, S. Tremaine, and E. Witten, Phys. Rev. D 95, 043541 (2017).

[66] H. Fukuda, S. Matsumoto, and T. T. Yanagida, Phys. Lett. B 789, 220 (2019). 